\title{
THE PHOTON SPECTRUM IN UPSILON SYSTEM DECAYS *
}

\author{
T. STERLING \\ Department of Physics, Unversity of Michigan, Ann Arbor, MI 48109
}

Received 26 May 1978

\begin{abstract}
The photon spectrum expected for the decays of the $\Upsilon$ system is calculated using the logarithmic potential model In order to estimate branching ratios, the leptonic and hadronic widths are estimated as well. The El branching ratios are found to be roughly double those for the $\psi$ system. This is largely due to the expected absence of hadronic cascades The purpose of the paper is to present the predictions of the quarkonium model for the entire photon spectrum in the $r$ region
\end{abstract}

\section{Introduction}

The new resonance found in proton + nucleus $\rightarrow \mu^{+} \mu^{-} \mathrm{X}[1]$ can be explained by the existence of a fifth quark. The two peaks, observed at $94 \mathrm{GeV}(\Upsilon)$ and 10.0 $\mathrm{GeV}\left(\Upsilon^{\prime}\right)$ would then be analogous to the $\psi$ and $\psi^{\prime}$. There may also be a third resonance at $10.4 \mathrm{GeV}\left(\Upsilon^{\prime \prime}\right)$. This system can be represented as two quarks bound by a potential. The $\Upsilon, \Upsilon^{\prime}$ and $\Upsilon^{\prime \prime}$ are the $1^{3} S, 2^{3} S$ and $3^{3} S$ states respectively We would also expect to find $\mathbf{P}$ and $\mathbf{D}$ states. Many features of the $\psi$-family of states can be reproduced using a linear-Coulomb potential [2]. A natural way to describe the $\Upsilon$ family would be to use the same potential as for the $\psi$ and increase the quark mass This was done by Eichten and Gottfried [3] prior to the discovery of the $\Upsilon$. For a quark mass of $5 \mathrm{GeV}$, Elchten and Gottfried predicted that the energy difference between $\Upsilon$ and $\Upsilon^{\prime}$ would be $420 \mathrm{MeV}$. This difference is observed to be about 600 $\mathrm{MeV}$ We can correct this problem by using a different potential for the $\Upsilon$.

The purpose of this paper is to investigate the photon spectrum expected from decayıng members of the $\Upsilon$ family. We use the logarithmic potential suggested by Quigg and Rosner [4] for all calculations The decay spectrum is expected to be dominated by electric dipole transitions and annihilations, both electromagnetic and strong. We will only consider the triplet states The magnetic dipole transitions and the decays $\Upsilon^{\prime} \rightarrow \Upsilon$ h hadrons which would populate the singlet state are expected to have small widths. Thus, the singlet states can be neglected in the first approximation. Furthermore, the hyperfine structure of the $\psi$-family is not a settled matter

\footnotetext{
\upported in part by the Department of Cnergy of the US.
} 
so it would be difficult to make reliable predictions for the singlet states. We will also assume that the binding energy is small relative to the quark masses so that $2 m \approx M, m$ being the quark mass and $M$ being the mass of the bound state in question.

Our main conclusions are the following. The El and leptonic decays for the $\Upsilon^{\prime}$ will have branching ratios about twice those for the $\psi^{\prime}$. This is largely due to the lack of hadronic cascades, which comprise about $50 \%$ of the $\psi^{\prime}$ width. The $\Upsilon^{\prime \prime}$ will have a rich spectrum which will be difficult to resolve The $\mathbf{P}$ and $\mathbf{D}$ states will have large El branching ratios, in some cases virtually $100 \%$

In sect. 2 we discuss possible potentrals and the masses of the bound states. Sect. 3 compares the logarithmic potential calculations with the $\psi$-data. The magnetic dipole transitions and hadronic cascades for the $\Upsilon$-system are discussed in sect. 4 Sects. 5, 6 and 7 present the formulae used to calculate the electric dipole transitions and the leptonic and hadronic decays

\section{Potentials, energy spacing and fine structure}

Some general features of the propertıes of particle-antıparticle bound states will be useful in selecting the potential for our calculations. The radial Schrodinger equation for two particles of equal mass, $m$, is

$$
-\frac{1}{m} \frac{\mathrm{d}^{2} u(r)}{\mathrm{d} r^{2}}+\left[\frac{l(l+1)}{m r^{2}}+V(r)-E\right] u(r)=0 .
$$

The wave function is

$$
\psi_{l m}(r, \Omega)=\frac{u(r)}{r} Y_{l m}(\Omega)=R(r) Y_{l m}(\Omega)
$$

where $u(r)$ has the normalization

$$
\int_{0}^{\infty}|u(r)|^{2} \mathrm{~d} r=1
$$

We can derive scaling rules for potentials in the form

$$
V(r)= \pm C r^{\nu}, \quad(- \text { for } \nu<0) .
$$

Substituting

$$
\begin{aligned}
& r=\rho(m C)^{-1 /(\nu+2)}, \\
& E=\xi m^{-\nu /(\nu+2)} C^{2 /(\nu+2),},
\end{aligned}
$$

into eq. (1) we have the dimensionless equation

$$
-\frac{\mathrm{d}^{2} w(\rho)}{\mathrm{d} \rho^{2}}+\left[\frac{l(l+1)}{\rho^{2}} \pm \rho^{\nu}-\xi\right] w(\rho)=0,
$$


where now

$$
\int_{0}^{\infty}|w(\rho)|^{2} \mathrm{~d} \rho=1,
$$

so

$$
w(\rho)=u\left(\rho(m C)^{-1 /(\nu+2)}\right)(m C)^{-1 /(2 \nu+4)},
$$

and

$$
R_{l}(\rho)=\frac{w(\rho)}{\rho}
$$

Results for the logarithmic potential,

$$
V(r)=C \ln (r) \text {, }
$$

will follow the scaling for $\nu=0$, except that $E$ is changed to

$$
E=C \xi-\ln \sqrt{m C},
$$

so that the energy differences do not change with $m$.

We can easily find the scaling rules for various decay rates. Electromagnetic (E1) transition rates scale by (see eq. (26)),

$$
\Gamma_{\mathrm{E} 1} \propto\left(E_{1}-E_{\mathrm{f}}\right)^{3}|\langle\mathrm{f}|r| \mathrm{i}\rangle|^{2} \propto m^{-(3 \nu+2) /(\nu+2)} .
$$

Annihilation rates for S-states are related to $m$ by (see eq. (28)),

$$
\Gamma \propto \frac{|R(0)|^{2}}{m^{2}} \propto m^{-(2 \nu+1) /(\nu+2)} .
$$

The fine structure correction terms are proportional to

$$
\frac{1}{m^{2}} \frac{\mathrm{d}^{2} V(r)}{\mathrm{d} r^{2}}, \frac{1}{m^{2} r} \frac{\mathrm{d} V(r)}{\mathrm{d} r} \propto m^{-(3 v+2) /(\nu+2)} .
$$

E1chten and Gottfried and others [2] have used a linear plus Coulomb potential,

$$
V(r)=\frac{4}{3} \frac{\alpha_{\mathrm{s}}}{r}+\frac{r}{a^{2}},
$$

to explain the behavior of the $\psi$ system. This form is motivated by the expected asymptotic behavior of the potential in color gauge theories. The extrapolation to the $\Upsilon$ predicts a $\Upsilon^{\prime}-\Upsilon$ mass splitting of $420 \mathrm{MeV}$ where $\sim 600 \mathrm{MeV}$ is measured. The $420 \mathrm{MeV}$ can be approximated just by scaling according to the linear potential $\left(E \propto m^{-1 / 3}\right)$ which dominates the potential in this region. This discrepancy and the fact that $\psi^{\prime}-\psi$ energy difference is also $\sim 600 \mathrm{MeV}$ led Quigg and Rosner [4] to try a logarithmic potential. Machacek and Tomozawa [5] had previously consl- 
dered logarithmic potentials to fit leptonic decay rates in the $\psi$. With a logarithmic potential the energy differences are independent of the quark mass (eq. (12)). The dımensionless Schrödınger equation is

$$
-\frac{\mathrm{d}^{2} w(\rho)}{\mathrm{d} \rho^{2}}+\left[\frac{l(l+1)}{\rho^{2}}+\ln \rho-\xi\right] w(\rho)=0 .
$$

The first few eigenvalues of this equation are listed in table 1.

There is no basic theoretical motivation for using the loganthmic potential ${ }^{\cdot}$ it is used because it is fairly simple and roughly fits the expermental data. Recently Celmaster and Henyey [6] have attempted to derive a potential from quantum chromodynamic theory. Their potential is similar to a logarithmic potential in the region where the wave function is large.

The average kinetic energy for the logarithmic potential is found from the virial theorem,

$$
\langle T\rangle=\left\langle\frac{r}{2} \frac{\mathrm{d} V}{\mathrm{~d} r}\right\rangle=\frac{1}{2} C,
$$

and is independent of the state. The speed is then given by $\langle\beta\rangle=\sqrt{\langle T\rangle / m}=\sqrt{C / 2 m}$. With $C=0.73 \mathrm{GeV}$ (to make $m\left(\Upsilon^{\prime}\right)-m(\Upsilon)=m\left(\psi^{\prime}\right)-m(\psi)=0.59 \mathrm{GeV}$ ) we have $\beta=0.47$ for $\psi(m=1.66 \mathrm{GeV})$ and $\beta=0.27$ for $\Upsilon(m=5 \mathrm{GeV})$. Although this is quite relativistic the results should be useful, at least qualitatively. The non-relatıvistic model has been used on systems much more relativistic than this. The $\Upsilon$ will be the closest to non-relativistic of the mesons we can presently study.

The fine structure terms for a logarithmic potential are proportional to $\mathrm{m}^{-1}$ so the ${ }^{3} \mathrm{P}$ splitting for the $\Upsilon$ should be a factor of 3 smaller than for the $\psi$. This would make the differences about $30 \mathrm{MeV}$ Although this small difference will make it diffi cult to distinguish the different energies of the P states experimentally, it can change E1 rates (e.g., $\Upsilon \rightarrow{ }^{3} \mathrm{P} \gamma$ ) by a factor of two (because of the $\left(E_{1}-E_{\mathrm{f}}\right)^{3}$ factor in eq. (26)).

Table 1

Energy eigenvalues for the log potential, eq. (13) and eq. (1)

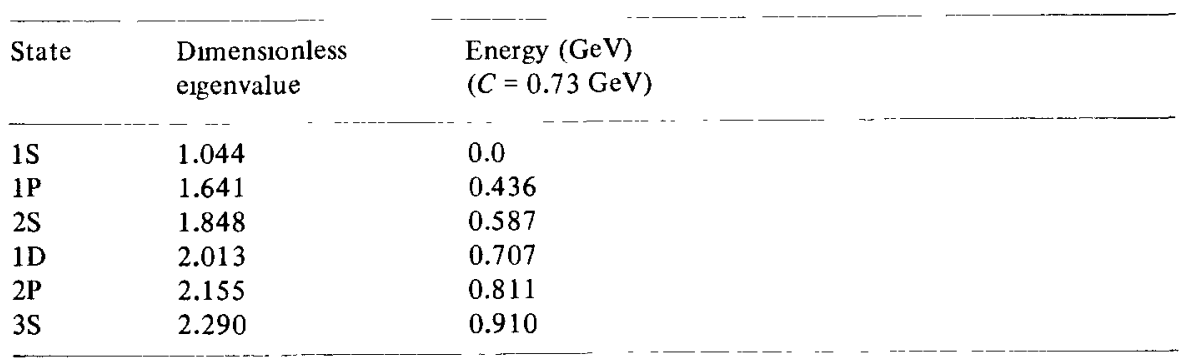


Table 2

Comparison of properties of $\psi$ particles with calculations using the linear Coulomb potential, $V(r)=-0.30 / r+r /(2.02 \mathrm{GeV})^{2}$, and the logarithmic potential $V(r)=(0.73 \mathrm{GeV}) \ln r+3.1$ $\mathrm{GeV}$.

\begin{tabular}{|c|c|c|c|c|c|}
\hline & Experim & ent $[7$ & & Lincar Coulomb $[1,3]$ & Logarithmic \\
\hline Mass of $1^{3} \mathrm{p}$ & 3522 & \pm 5 & $\mathrm{MeV}$ & 3457 & 3531 \\
\hline$\left|\psi_{2 S}(0) / \psi_{1 S}(0)\right|^{2}$ & 0.62 & \pm 0.16 & & 0.91 & 051 \\
\hline$\Gamma(\psi \rightarrow \ell \bar{\ell})$ & 4.8 & \pm 0.6 & $\mathrm{keV}$ & 5.3 & 88 \\
\hline$\Gamma\left(\psi^{\prime} \rightarrow{ }^{3} \mathrm{P}_{2} \gamma\right)$ & 16 & \pm 9 & $\mathrm{keV}$ & 27 & 36 \\
\hline$\Gamma\left(\psi^{\prime} \rightarrow{ }_{3}^{3} \mathrm{P}_{1} \gamma\right)$ & 16 & \pm 8 & $\mathrm{keV}$ & 38 & 50 \\
\hline$\Gamma\left(\psi^{\prime} \rightarrow{ }^{3} \mathrm{P}_{0} \gamma\right)$ & 16 & \pm 9 & $\mathrm{keV}$ & 44 & 58 \\
\hline
\end{tabular}

\section{Comparison of theory and data for the $\Psi$ family}

The reliability of predictions made for the $\Upsilon$ family with the log potential can be gauged by the degree of success of the analogous calculations for the $\psi$. Some predictions one obtains for the $\psi$ family decays calculated using the log potential are compared to those from the linear-Coulomb potential and to experiment in table 2. The potentials used were fitted to the energies of the $1^{3} \mathrm{~S}$ and $2^{3} \mathrm{~S}$ states $\left(\psi\right.$ and $\left.\psi^{\prime}\right)$, giving $\alpha_{\mathrm{s}}=023$ and $a=2.07 \mathrm{GeV}$ for the linear-Coulomb potential (eq. (16)) and $C=0.73 \mathrm{GeV}$ for the logarithmic potential (eq. (11)). The E1 rates were calculated using the measured energy differences for the $\left(E_{\mathrm{i}}-E_{\mathrm{f}}\right)^{3}$ factor in eq. (26) and the calculated overlap integrals for the $\left\langle w_{\mathbf{f}}|\rho| w_{1}\right\rangle$ factor. The measured $1^{3} \mathrm{P}$ energies $[7,8]$ and the $2^{3} \mathrm{~S}_{1}$ to $1^{3} \mathrm{~S}_{1}$ wave function ratio at the origin (from measurements made by Luth et al. [9] and Bayarski et al. [10]) are in good agreement with the calculations. However, the calculated E1 transition rates are too high by a factor of 2 or 3 . This is a persistent problem in the non-relativistic potential model of the $\psi$. More detaled calculations takıng into account the charmed meson continuum, done by E1chten et al. [11] achieve good agreement for some transitions but are still off by a factor of two or three for others.

\section{M1 transitions and hadronic cascades}

The transition rate for allowed magnetic dipole (M1) transitions is

$$
\Gamma_{\text {allowed }}=\frac{e_{\mathrm{Q}}^{2} \alpha k^{3}}{3 m^{2}}\left(2 J_{\mathrm{f}}+1\right)
$$

where $k$ is the energy difference between the triplet and singlet states and we have assumed the radial wave functions to be identical. If the splitting between the trip- 
let and singlet states were due to the normal hyperfine splittıng process then

$$
k \propto m^{-1}
$$

so

$$
\Gamma_{\text {allowed }} \propto m^{-5} \text {. }
$$

If we then use this to scale from the $\psi-\eta_{\mathrm{c}}$ (assuming that the $\eta_{\mathrm{c}}(2900)$ is the $1^{1} \mathrm{~S}_{0}$ state) then $[7,10]$

$$
\Gamma_{\text {allowed }}\left(\Upsilon\left(1^{3} \mathrm{~S}_{1}\right) \rightarrow 1^{1} \mathrm{~S}_{0}\right) \leqslant \frac{1.2 \mathrm{keV}}{3^{5}} \approx 5 \mathrm{eV}
$$

This is much smaller than any other decays and so will be neglected. It should be noted that the formula for the transition rate works very badly for the $\psi-\eta_{\mathrm{c}}$, the calculated rate is $29 \mathrm{keV}$ where the observed rate is $\lesssim 1.2 \mathrm{keV}[7,10]$.

There are also strong interaction transitions which can create the $1^{1} \mathrm{~S}_{0}$ state, for example

$$
1^{3} \mathrm{P}_{1} \rightarrow 1^{1} \mathrm{~S}_{0}+2 \pi
$$

It can be shown that because of the strength of the $\Upsilon^{\prime}$ signal relative to the $\Upsilon$ signal (in $p+$ nucleus $\rightarrow \mu^{+} \mu^{-} X$ ) there cannot be a large rate for $\Upsilon^{\prime} \rightarrow \Upsilon+2 \pi$. We then assume that this is because of a decoupling of the light hadrons from the new quark and claim that all such hadronic transitions are small. The argument that $\Upsilon^{\prime} \rightarrow \Upsilon+2 \pi$ is suppressed was given by Cahn and Ellis [12] who also gave a possible alternative explanation, that there are actually two new quarks and the $\Upsilon$ and $\Upsilon^{\prime}$ are not $1^{3} S_{1}$ and $2^{3} S_{1}$ states. The ratio of the $\mu^{+} \mu^{-}$signal for $\Upsilon^{\prime}$ to that for $\Upsilon$ is about 0.36 . Let $\sigma_{\Upsilon}$ and $\sigma_{\Upsilon}$ be the total production cross sections for $\Upsilon$ and $\Upsilon^{\prime}$ respectively, not including $\Upsilon$ 's and $\Upsilon$ 's produced by decays of other members of the $\Upsilon$ family. The $\mu^{+} \mu^{-}$signal ratio, $R$ is given by

$$
R=\frac{\sigma_{\Upsilon^{\prime} \mathrm{BR}\left(\Upsilon^{\prime} \rightarrow \mu^{+} \mu^{-}\right)}}{\left(\sigma_{\Upsilon}+\sigma_{\Upsilon^{\prime}} \mathrm{BR}\left(\Upsilon^{\prime} \rightarrow \Upsilon \mathrm{X}\right)\right) \mathrm{BR}\left(\Upsilon \rightarrow \mu^{+} \mu^{-}\right)} .
$$

Cahn and Ellıs, using estımates for the cross sections and branching ratios, find an upper bound for $\Gamma\left(\Upsilon^{\prime} \rightarrow \Upsilon h\right.$ ) where $h$ is any light hadronic system (e.g. $\pi \pi$ or $\eta$ )

$$
\begin{aligned}
\Gamma\left(\Upsilon^{\prime} \rightarrow \Upsilon \mathrm{h}\right) & \lesssim 1 \mathrm{keV} & & \text { for } e_{\mathrm{Q}}-\frac{1}{3} \\
& \lesssim 5 \mathrm{keV} & & \text { for } e_{\mathrm{Q}}=\frac{2}{3} .
\end{aligned}
$$

These numbers are somewhat dependent on the estimates made but even with drastic changes the w1dth is small $(\leqslant 10 \mathrm{keV})$.

In another approach Gottfried [13] has shown a multipole expansion of the color gauge field and claims that asymptotically (with large $m$ ) the two-pion transition $\Upsilon^{\prime} \rightarrow \Upsilon+2 \pi$ would vary like $m^{-2}$. If this can be used to scale from the $\psi$ then $\Gamma\left(\Upsilon^{\prime} \rightarrow \Upsilon+2 \pi\right) \approx 10 \mathrm{keV}$ 
All hadronic cascades may not be small as is assumed in this paper. Aside from the $\Upsilon^{\prime} \rightarrow \Upsilon$ transitions there is no evidence either way. In the case they are not small the results must be modified to take them into account.

\section{E1 transition rates for $\Upsilon$}

Radiatıve transitıons can be calculated using the usual non-relativistıc rate formula

$$
\Gamma=\frac{4}{3} \alpha e_{\mathrm{Q}}^{2}\left(E_{1}-E_{\mathrm{f}}\right)^{3}(2 \jmath+1) S_{\mathrm{fi}}\left|\left\langle n l|r| n^{\prime} l^{\prime}\right\rangle\right|^{2} \text {. }
$$

Table 3

Behavior of dimensionless wave functions from eq. (17) at the origin $\left(R_{l}\right.$ is defined in eq (10))

\begin{tabular}{lccccc}
\hline State & $R_{l}(0)$ & State & $R_{l}^{\prime}(0)$ & State & $R_{l}^{\prime \prime}(0)$ \\
\hline 1S & 0.830 & $1 \mathrm{P}$ & 0.245 & $1 \mathrm{D}$ & 0.122 \\
2S & 0.593 & $2 \mathrm{P}$ & 0.240 & & \\
$3 \mathrm{~S}$ & 0.490 & & & & \\
\hline
\end{tabular}

Table 4

Geometric factor for E1 transitions (eq. (27))

\begin{tabular}{lccc} 
For $^{3} \mathrm{P}_{\mathrm{J}} \rightarrow{ }^{3} \mathrm{D}_{\mathrm{J}}$ & $J$ & $J^{\prime}$ & $S_{\mathrm{fi}}$ \\
\hline & 0 & 1 & $2 / 9$ \\
& 1 & 1 & $1 / 18$ \\
1 & 2 & $1 / 10$ \\
2 & 1 & $1 / 450$ \\
2 & 2 & $1 / 50$ \\
2 & 3 & $2 / 25$
\end{tabular}

For ${ }^{3} \mathrm{~S}_{1} \rightarrow{ }^{3} \mathrm{P}_{\mathrm{J}}, S_{\mathrm{f}_{\mathrm{l}}}=\frac{1}{3}$. For arbitrary transitions $S_{\mathrm{f} \mathbf{l}}=\left\{\begin{array}{l}l_{1} l^{\prime} \\ l^{\prime} l\end{array}\right\}^{2} \max \left(l, l^{\prime}\right)$ with $l l s \rightarrow j^{\prime} l^{\prime} s[18]$. Note that $S_{\mathrm{f} \mathbf{t}}=S_{\mathrm{if}}$.

Table 5

Dimensionless radial overlap integrals required for E1 transitions ( $w$ is defined in eq. (9))

\begin{tabular}{lcc}
$\left\langle w_{2}\right|$ & $\left|w_{1}\right\rangle$ & $\left\langle w_{2}|\rho| w_{1}\right\rangle$ \\
\hline $1 S$ & $1 \mathrm{P}$ & 2.15 \\
$1 \mathrm{~S}$ & $2 \mathrm{P}$ & 0.41 \\
$1 \mathrm{P}$ & $1 \mathrm{D}$ & 3.56 \\
$1 \mathrm{P}$ & $2 \mathrm{~S}$ & -2.90 \\
$1 \mathrm{P}$ & $3 \mathrm{~S}$ & -0.10 \\
$2 \mathrm{~S}$ & $2 \mathrm{P}$ & 3.76 \\
$2 \mathrm{P}$ & $1 \mathrm{D}$ & -3.53 \\
$2 \mathrm{P}$ & $3 \mathrm{~S}$ & -5.03 \\
\hline
\end{tabular}


For the $\log$ potential we have

$$
\Gamma=\frac{4}{3} \alpha e_{\mathrm{Q}}^{2} \frac{C^{2}}{m}\left(\xi_{1}-\xi_{\mathrm{f}}\right)^{3}(2 j+1) S_{\mathrm{fi}}\left|\left\langle w|\rho| w^{\prime}\right\rangle\right|^{2}
$$

for an E1 transition from a state with $j^{\prime} l^{\prime} s$ going to a state with $j l s . e_{\mathrm{Q}}$ is the quark charge in units of the electron's charge, $\alpha$ the fine structure constant and $S_{\mathrm{fi}}$ is a geometric factor. The $S_{\mathrm{fi}}$ factors are tabulated in table 4 and the dimensionless radial dipole overlap integrals $\left\langle w|\rho| w^{\prime}\right\rangle$, are in table 5 .

In table 2 we see that for the log potential the predicted $\mathrm{E} 1$ rates are about three times larger than the observed rates for $\psi^{\prime} \rightarrow{ }^{3} \mathrm{P} \psi$. The theoretical rates were found using the same radial wave functions for all the $1^{3} \mathrm{P}$ states. This discrepancy ind1cates that the analogous calculations for the $\Upsilon$ may also be high by a factor of two or three.

\section{Leptonic decays}

The ${ }^{3} S_{1}$ states can decay electromagnetically into a lepton pair. The rate for this process is [14]

$$
\Gamma_{Q^{+} \ell^{-}}=\frac{\alpha^{2} e_{\mathrm{Q}}^{2}|R(0)|^{2}}{m^{2}}
$$

For the $\log$ potential, eq. (11), we have

$$
\Gamma_{\ell^{+} \ell^{-}}=\alpha^{2} e_{\mathrm{Q}}^{2}\left(\frac{C}{m}\right)^{3 / 2} m\left|R_{l}(0)\right|^{2},
$$

where $e_{\mathrm{Q}}$ is the quark charge. The ${ }^{3} \mathrm{P}$ states are forbidden to decay to a fermion and an anti-fermion via a virtual photon but the ${ }^{3} \mathrm{D}_{1}$ may decay this way. Direct calculation of the ${ }^{3} \mathrm{D}_{1}$ state decay is difficult because $1 \mathrm{t}$ is due to second order relativistic corrections. By dımensional arguments we find

$$
\Gamma \propto \alpha^{2} e_{\mathrm{Q}}^{2} \frac{\left|R^{\prime \prime}(0)\right|^{2}}{m^{6}},
$$

and for the $\log$ potential

$$
\Gamma \propto \alpha^{2} e_{\mathrm{Q}}^{2}\left(\frac{C}{m}\right)^{7 / 2} m\left|R_{l}^{\prime \prime}(0)\right|^{2} \approx 6 \mathrm{eV},
$$

which is quite small. Some properties of the wave functions at the origin given by the log potential are given in table 3 .

\section{Hadronic decays}

Annihilation into hadrons can be calculated by assuming the color gauge theory for the quarks, calculating the widths to free quarks and gluons and assuming the 
free quarks and gluons turn into hadrons without influencing the rate. The triplet $\mathrm{S}$ states can annihilate into three gluons. Except for the color factor the problem is the same as the analogous decay of orthopositronium into three photons [15]. The rate is

$$
\Gamma_{\mathrm{had}}=\frac{40}{81 \pi}\left(\pi^{2}-9\right) \frac{\alpha_{\mathrm{s}}^{3}}{(2 m)^{2}}|R(0)|^{2} .
$$

For the $\log$ potential, we have

$$
\Gamma_{\mathrm{had}}=\frac{10}{81 \pi}\left(\pi^{2}-9\right) \alpha_{\mathrm{s}}^{3}\left(\frac{C}{m}\right)^{3 / 2} m\left|R_{l}(0)\right|^{2}
$$

where $\alpha_{\mathrm{s}}$ is related to the strong coupling constant $g,\left(\alpha_{\mathrm{s}}=g^{2} / 4 \pi\right)$. S-states can also decay electromagnetically into two quarks by the same mechanism by which they decay into two leptons. The leptonic rate is multiplied by a color factor of three and a factor $e_{\mathrm{Q} 1}^{2} e_{\mathrm{Q} 2}^{2}(\mathrm{Q} 1$ decay1ng into $\mathrm{Q} 2)$ because the quarks may have fractional charge. If we sum over two quatk flavo1s with $e_{\mathrm{Q} 2}=-\frac{1}{3}$ and two with $e_{\mathrm{Q} 2}=\frac{2}{3}$ we have for the logarithmic potential

$$
\Gamma_{\gamma}^{* \rightarrow h}=\frac{10}{3} \Gamma_{\ell^{+} \ell^{-}} \text {. }
$$

The masses of the lighter quarks are taken to be zero which may not be a good approximation for the charmed quark.

For the P-states, the $0^{++}$and $2^{++}$states decay into 2 gluons while the largest contribution to the $1^{++}$decay is to one gluon and a quark pair [17]. The rate for $0^{++}$and $2^{++}$was calculated by Barbier1, Gatto and Kogerler [16] to be

$$
\begin{aligned}
& \Gamma_{0^{++}}=\frac{96 \alpha_{\mathrm{s}}^{2}}{(2 m)^{4}}\left|R^{\prime}(0)\right|^{2}=6 \alpha_{\mathrm{s}}^{2}\left(\frac{C}{m}\right)^{5 / 2} m\left|R_{l}^{\prime}(0)\right|^{2}, \\
& \Gamma_{2^{++}}=\frac{128}{5} \frac{\alpha_{\mathrm{s}}^{2}}{(2 m)^{4}}\left|R^{\prime}(0)\right|^{2} \\
& \quad=\frac{8}{5} \alpha_{\mathrm{s}}^{2}\left(\frac{C}{m}\right)^{5 / 2} m\left|R_{l}^{\prime}(0)\right|^{2}
\end{aligned}
$$

The $1^{++}$rate was found by Barbierı, Gatto and Remidd [17] to be

$$
\begin{aligned}
\Gamma_{1++} & =\frac{n}{3} \frac{256}{3 \pi}-\frac{\alpha_{\mathrm{s}}^{3}}{(2 m)^{4}}\left|R^{\prime}(0)\right|^{2} \ln \frac{1}{\alpha_{\mathrm{s}}} \\
= & \frac{n}{3} \frac{16}{3 \pi} \alpha_{\mathrm{s}}^{3} \ln \frac{1}{\alpha_{\mathrm{s}}}\left(\frac{C}{m}\right)^{5 / 2} m\left|R_{l}^{\prime}(0)\right|^{2},
\end{aligned}
$$

where $n$ is the number of quark flavors with a mass smaller than $m$ (for $\Upsilon, n=4$ ). To arrive at this form they had to approximate the binding energy. The binding 
energy was estimated using

$$
\frac{4 m^{2}-M}{M^{2}} \approx \alpha_{\mathrm{s}}^{2}
$$

which should be a good approximation for large masses.

The ${ }^{3} \mathrm{D}_{1}$ state rates have not been calculated because of the difficulties discussed in the leptonic decays (sect. 5). However, we know that two gluon decays are forbidden so the rate will be in the form

$$
\Gamma=N \alpha_{\mathrm{s}}^{3}\left(\frac{C}{m}\right)^{7 / 2} m\left|R_{l}^{\prime \prime}(0)\right|^{2} \approx N(03 \mathrm{keV}),
$$

where $N$ is a numerical constant. This rate is small if $N$ is of order unity.

\section{Summary}

The formulae in the preceeding sections have been used to calculate the expected decay rates for the $\Upsilon$ family. The rates for a quark mass of $5 \mathrm{GeV}$ and both $\frac{2}{3}$ and $-\frac{1}{3}$ charge are given in table 6 . The logarithmic potentıal with $C=0.73 \mathrm{GeV}$ to fit the $\Upsilon^{\prime}-\Upsilon$ mass difference was used. The branching ratios are included assuming there are no other significant decays. Transitions between members of the $\Upsilon$ family emitting light hadrons are omitted, because the present data indicate they have small widths. This indicates that experimenters must be prepared to find rather small rates of pion emission. The E1 rates calculated here can be expected to be higher by a factor of 2 or 3 than what we can reasonably expect to see. If it is true that the suppression of pionic transitions is due to a general decoupling of the new quarks to old quarks then the rates for annihilation into hadrons may also be lower than the calculations.

To get the total spectrum of photons in $\mathrm{e}^{+} \mathrm{e}^{-}$annihilation cascades must be counted. For instance, for $e_{\mathrm{Q}}=-\frac{1}{3}$ the $\Upsilon^{\prime}$ has a widths of $8.5 \mathrm{keV}$ to decay to the $1 P$ states emitting a $151 \mathrm{MeV}$ photon, with a branching ratio of $36 \%$. About $16 \%$ of these $1^{3} \mathrm{P}$ states will then emit a $436 \mathrm{MeV}$ photon, so we have a $436 \mathrm{MeV}$ photon for $6 \%$ of the original $\Upsilon^{\prime}$. If the fine structure split ting is large enough these will be split into three peaks. When the E1 rates are modified to adjust for the overestimated E1 rates in potential models, $15 \%$ of the $\Upsilon^{\prime}$ decay to $1 \mathrm{P}$ states, with only $2 \%$ of the original $\Upsilon^{\prime}$ producing $\Upsilon$.

The spectrum for the $\Upsilon^{\prime \prime}$ is much more complicated than that for the $\Upsilon^{\prime}$. There are 29 different transitions with photon energies ranging from about $70 \mathrm{MeV}$ to $850 \mathrm{MeV}$, as shown in fig. 2. This spectrum will be quite difficult to sort out, as it has many close lines. The P-state energy splittings shown in fig. 2 were scaled from the $\psi$ system and the D states were split just to show the separate lines. Although these energies are only rough estımates, it is clear that very good energy resolution will be necessary to resolve these lines 
Table 6

Decay rates for the $\Upsilon$ family for the logarithmic potential $V(r)=0.73 \mathrm{GeV} \ln r$.

State $\left(J^{P C}\right) \quad$ Final state $\quad q=-\frac{1}{3} \quad q=$

\begin{tabular}{|c|c|c|c|c|c|}
\hline & & & \\
\hline & & $\Gamma(\mathrm{keV})$ & $\mathrm{BR}(\%)$ & $\Gamma(\mathrm{keV})$ & $\mathrm{BR}(\%)$ \\
\hline $3^{3} s_{1}\left(1^{--}\right)$ & $\begin{array}{l}2^{3} \mathrm{P}_{0}\left(0^{++}\right) \\
2^{3} \mathrm{P}_{1}\left(1^{++}\right) \\
2{ }^{3} \mathrm{P}_{2}\left(2^{++}\right) \\
\text {hadrons (strong) } \\
\text { hadrons (e.m.) } \\
\text { leptons } \\
\text { total }\end{array}$ & $\begin{array}{l}0.80 \\
2.4 \\
4.0 \\
7.7 \\
1.3 \\
1.2 \\
17.4\end{array}$ & $\begin{array}{c}5(2) \\
14(6) \\
23(14) \\
44(59) \\
7(10) \\
7(9)\end{array}$ & $\begin{array}{r}3.2 \\
9.5 \\
15.9 \\
7.7 \\
5.3 \\
4.8 \\
46.5\end{array}$ & $\begin{array}{c}7(3) \\
20(11) \\
34(25) \\
17(27) \\
11(18) \\
10(17)\end{array}$ \\
\hline $2^{3} \mathrm{P}_{0}^{++}$ & $\begin{array}{l}1^{3} \mathrm{D}_{1}-- \\
2^{3} \mathrm{~S}_{1}-- \\
1^{3} \mathrm{~S}_{1}-- \\
\text { hadrons (strong) } \\
\text { total }\end{array}$ & $\begin{array}{r}2.7 \\
15.7 \\
8.8 \\
319 \\
346\end{array}$ & $\begin{array}{c}0.8(0.3) \\
5(1) \\
3(0.7) \\
92(98)\end{array}$ & $\begin{array}{l}10.9 \\
62.7 \\
35.3 \\
319 \\
428\end{array}$ & $\begin{array}{c}2(1) \\
15(5) \\
8(3) \\
75(91)\end{array}$ \\
\hline $2^{3} \mathrm{P}_{1}++$ & $\begin{array}{l}1^{3} \mathrm{D}_{1}-- \\
1^{3} \mathrm{D}_{2}-- \\
2^{3} \mathrm{~S}_{1} \\
1^{3} \mathrm{~S}_{1} \\
\text { hadrons (strong) } \\
\text { total }\end{array}$ & $\begin{array}{r}0.7 \\
2.1 \\
157 \\
8.8 \\
34.3 \\
61.6\end{array}$ & $\begin{array}{c}1(0.3) \\
3(1) \\
25(33) \\
14(18) \\
56(47)\end{array}$ & $\begin{array}{r}2.7 \\
8.2 \\
62.7 \\
35.3 \\
34.3 \\
143.2\end{array}$ & $\begin{array}{c}2(0.5) \\
6(2) \\
44(51) \\
25(29) \\
24(18)\end{array}$ \\
\hline $2^{3} \mathrm{P}_{2}^{++}$ & $\begin{array}{l}1^{3} \mathrm{D}_{1}-- \\
1^{3} \mathrm{D}_{2--} \\
1^{3} \mathrm{D}_{3}^{--} \\
2^{3} \mathrm{~S}_{1--} \\
1^{3} \mathrm{~S}_{1}-- \\
\text { hadrons (strong) } \\
\text { total }\end{array}$ & $\begin{array}{r}0.03 \\
0.4 \\
2.3 \\
15.7 \\
8.8 \\
85.1 \\
112.3\end{array}$ & $\begin{array}{l}0.02(0.01) \\
0.4(0.16) \\
2(1) \\
14(8) \\
8(4) \\
76(87)\end{array}$ & $\begin{array}{r}0.1 \\
1.6 \\
9.2 \\
62.7 \\
35.3 \\
85.1 \\
144.0\end{array}$ & $\begin{array}{l}0.05(0.03) \\
0.8(0.5) \\
5(3) \\
32(22) \\
18(12) \\
44(62)\end{array}$ \\
\hline $1^{3} D_{1}--$ & $\begin{array}{l}1^{3} \mathrm{P}_{0^{++}} \\
1^{3} \mathrm{P}_{1}^{++} \\
1^{3} \mathrm{P}_{2}^{++} \\
\text {hadrons (strong) } \\
\text { hadrons (em) } \\
\text { leptons } \\
\text { total }\end{array}$ & $\begin{array}{c}16.7 \\
12.5 \\
0.8 \\
\mathrm{~N}(0.3) \\
\mathrm{N}^{\prime}(0.007) \\
\mathrm{N}^{\prime}(0.006) \\
30.0^{+}\end{array}$ & $\begin{array}{c}56(56) \\
42(42) \\
3(3)\end{array}$ & $\begin{array}{l}66.7 \\
50.0 \\
3.3 \\
\mathrm{~N}(0.3) \\
\mathrm{N}^{\prime}(0.007) \\
\mathrm{N}^{\prime}(0.006) \\
120.0+\end{array}$ & $\begin{array}{c}56(56) \\
42(42) \\
3(3)\end{array}$ \\
\hline $1^{3} \mathrm{D}_{2}--$ & $\begin{array}{l}1^{3} \mathrm{P}_{1}- \\
1{ }^{3} \mathrm{P}_{2}- \\
\text { hadrons (strong) } \\
\text { total }\end{array}$ & $\begin{array}{r}22.5 \\
7.5 \\
\mathrm{~N}(0.3) \\
30.0+\end{array}$ & $\begin{array}{l}75(75) \\
25(25)\end{array}$ & $\begin{array}{c}90.0 \\
30.0 \\
\mathrm{~N}(0.3) \\
120.0^{+}\end{array}$ & $\begin{array}{l}75(75) \\
25(25)\end{array}$ \\
\hline $1^{3} \mathrm{D}_{3}--$ & $\begin{array}{l}1^{3} \mathrm{P}_{2}-- \\
\text { hadrons (strong) } \\
\text { total }\end{array}$ & $\begin{array}{c}30.0 \\
N(0.3) \\
30.0+\end{array}$ & $100(100)$ & $\begin{array}{l}120.0 \\
\mathrm{~N}(0.3) \\
120.0+\end{array}$ & $100(100)$ \\
\hline
\end{tabular}


Table 6 (continued)

\begin{tabular}{|c|c|c|c|c|c|}
\hline \multirow[t]{7}{*}{$2^{3} S_{1}--$} & $1^{3} \mathrm{P}_{0^{++}}$ & 1.0 & $4(1)$ & 3.8 & $6(3)$ \\
\hline & $1^{3} \mathrm{P}_{1}^{++}$ & 2.9 & $12(4)$ & 11.4 & $19(9)$ \\
\hline & $1^{3} \mathrm{P}_{2}^{++}$ & 4.8 & $20(10)$ & 19.1 & $32(22)$ \\
\hline & hadrons (strong) & 11.3 & $48(54)$ & 11.3 & $19(29)$ \\
\hline & hadrons $(\mathrm{em})$ & 1.9 & $8(9)$ & 7.7 & $13(20)$ \\
\hline & leptons & 1.8 & $8(9)$ & 7.0 & $12(18)$ \\
\hline & total & 23.6 & & 60.3 & \\
\hline \multirow[t]{3}{*}{${ }_{1}^{3} \mathrm{P}_{0}++$} & $1^{3} S_{1}$ & 12.6 & $4(1)$ & 50.3 & $13(4)$ \\
\hline & hadrons (strong) & 330.0 & $96(94)$ & 330.0 & $87(96)$ \\
\hline & total & 342.6 & & & \\
\hline \multirow[t]{3}{*}{$1^{3} \mathrm{P}_{1}^{++}$} & $1^{3} \mathrm{~S}_{1}$ & 12.6 & $26(35)$ & 50.3 & $59(68)$ \\
\hline & hadrons (strong) & 35.9 & $74(65)$ & 35.9 & $41(32)$ \\
\hline & total & 48.0 & & 85.7 & \\
\hline \multirow[t]{3}{*}{$1^{3} \mathrm{P}_{2}^{++}$} & $1^{3} S_{1}$ & 12.6 & $13(6)$ & 50.3 & $36(22)$ \\
\hline & hadrons (strong) & 88.0 & $87(94)$ & 88.0 & $64(78)$ \\
\hline & total & 100.6 & & 138.3 & \\
\hline \multirow[t]{4}{*}{$1^{3} S_{1}--$} & hadrons (strong) 1 & 22.2 & 76 & 22.2 & 43 \\
\hline & hadrons (em) & 3.4 & 12 & 13.8 & 27 \\
\hline & leptons & 3.8 & 13 & 15.2 & 30 \\
\hline & total & 29.4 & & 51.2 & \\
\hline
\end{tabular}

The $\mathrm{E} 1$ ratios are expected to be too high by a factor of two or three by analogy to similar calculations for the $\psi$ family. In parentheses branching ratios are given using a subjective correction factor taken from the charmonium spectrum, if the reason for the discrepancies with exper1ment for charmonium also hold for the $\Upsilon$ then the numbers in parentheses will be more realistic. $N$ and $N^{\prime}$ are unknown constants, considered to be small.

The most likely decay chans for $\Upsilon^{\prime \prime}$ are $\Upsilon\left(3^{3} S_{1}\right) \rightarrow$ hadrons (28\%) (51\%) and $\Upsilon\left(3^{3} \mathrm{~S}_{1}\right) \rightarrow \gamma \Upsilon\left(2^{3} \mathrm{P}_{2}\right), \Upsilon\left(2^{2} \mathrm{P}_{2}\right) \rightarrow$ hadrons $(19 \%)(17 \%)$. The chain $\Upsilon\left(3^{2} \mathrm{~S}_{1}\right) \rightarrow$ $\gamma \Upsilon\left(2^{3} \mathrm{P}_{2}\right), \Upsilon\left(2^{3} \mathrm{P}_{2}\right) \rightarrow \gamma\left(2^{3} \mathrm{~S}_{1}\right), \Upsilon\left(2^{3} \mathrm{~S}_{1}\right) \rightarrow \gamma \Upsilon\left(1^{3} \mathrm{P}_{2}\right) \rightarrow \gamma \Upsilon\left(1^{3} \mathrm{~S}_{1}\right)$ has a probability of $1.3 \%(0.1 \%)$ for $q=+\frac{2}{3}\left(-\frac{1}{3}\right)$. The D-states decay virtually entırely by $\mathrm{E} 1$ but are only created for about $4 \%$ of the $\Upsilon^{\prime \prime}$. Most of the $\Upsilon$ particles created will come from the $\Upsilon\left(2^{3} \mathrm{P}\right) \rightarrow \Upsilon\left(1^{3} \mathrm{~S}_{1}\right)$ decays.

In proton + nucleus experiments the production of $\Upsilon$ has a contribution from production of higher energy states which then emit photons producing $\Upsilon$. A rough approximation of the production scheme is to assume that all bound states in the $\Upsilon$ system are produced at the same rate from the initial reaction. The total production can then be calculated from the branching ratios. If the initial production rate of each state is 1 , then the total production of $\Upsilon$ is 2.3 and the total production of $\Upsilon^{\prime}$ is 1.5 assuming $e_{\mathrm{Q}}=-\frac{1}{3}$. We see that some $56 \%$ of the $\Upsilon$ and $33 \%$ of the $\Upsilon^{\prime}$ originate at higher levels. Using the branchung ratios for decay into leptons we can find 

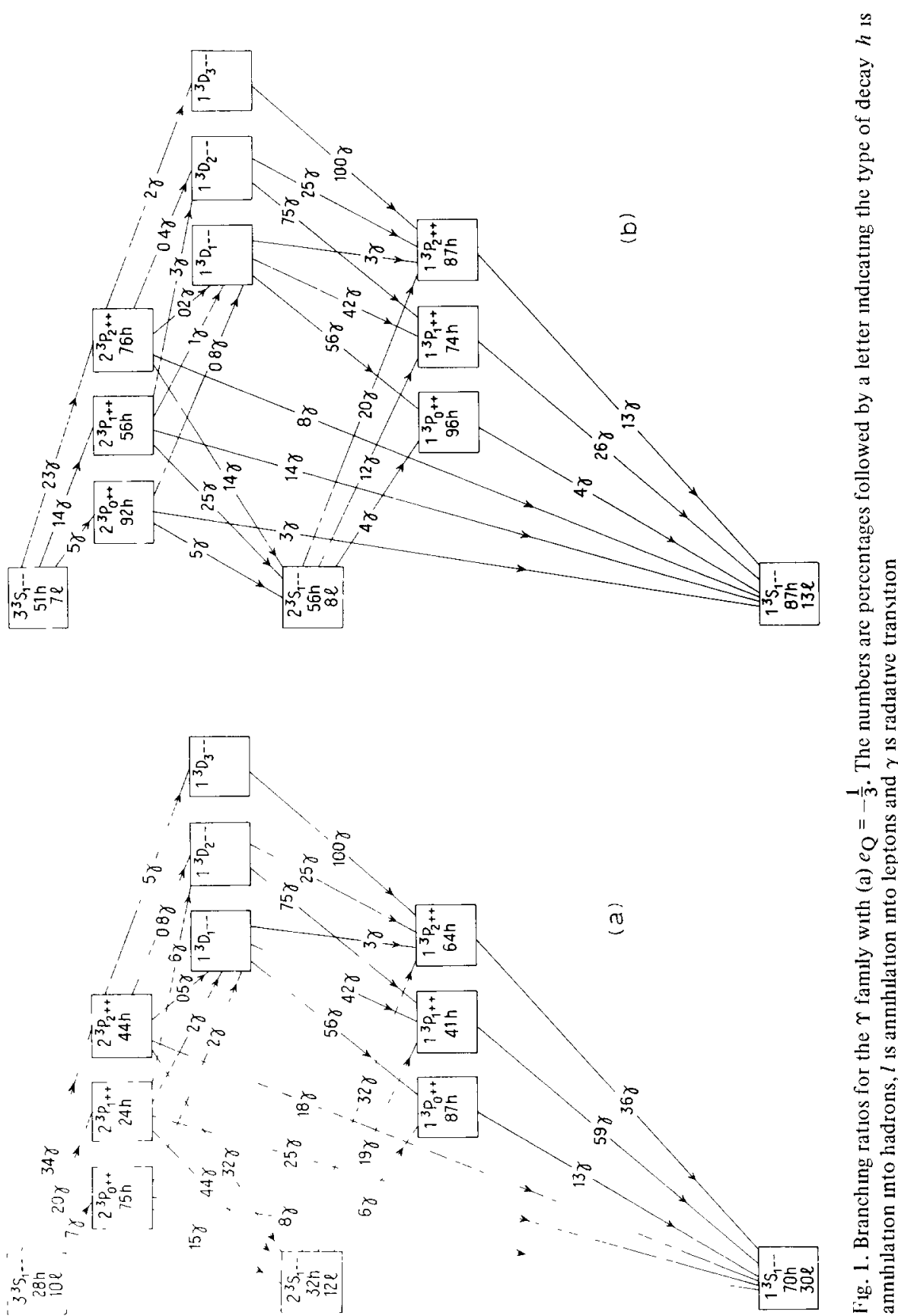

心in
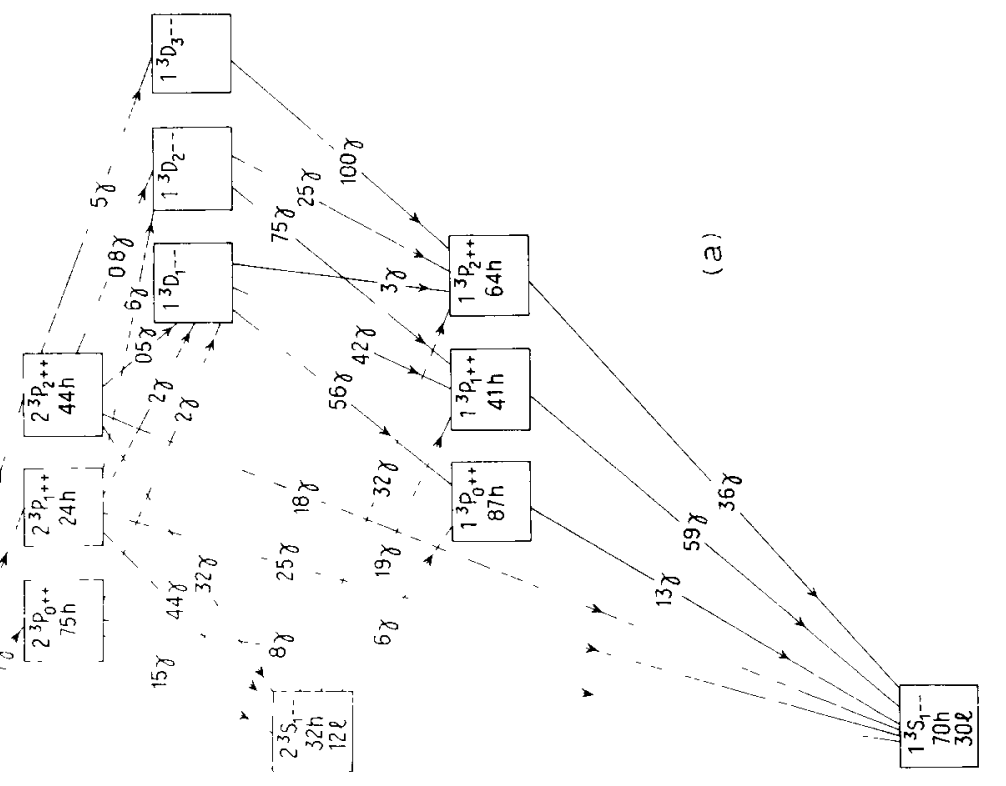


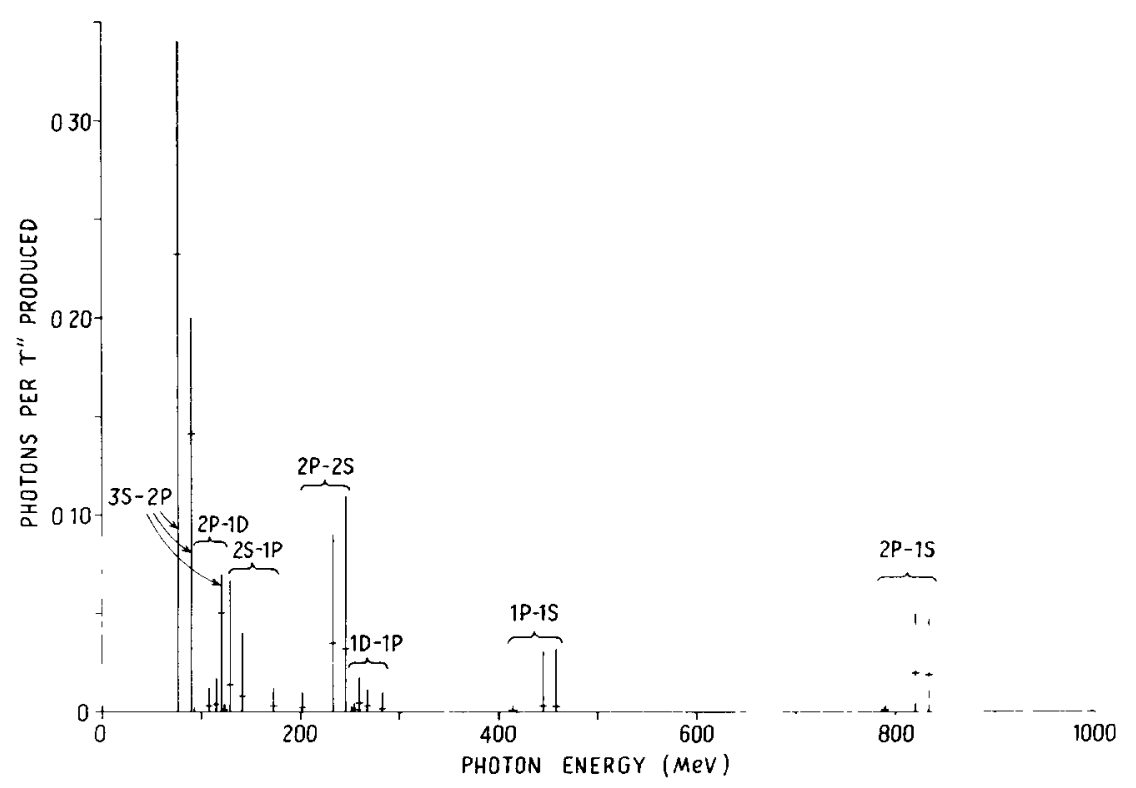

Fig. 2. The intensity of photons as a function of energy for $e_{\mathrm{Q}}=\frac{2}{3}$ and $-\frac{1}{3}$ (The intensity of $e_{\mathrm{Q}}=$ $-\frac{1}{3}$ is indicated by a small line crossing each main line.) The P-state splitting shown was found by scaling from the $\psi$ system. These energy differences were not used in calculating the widths. A small splittıng is shown for the D states only to separate the lines.

the ratio of leptons from the $\Upsilon^{\prime}$ and $\Upsilon$ to be

$$
R=0.40 \text {. }
$$

In good agreement with the experimentally measured 0.36 . Using $e_{\mathrm{Q}}=\frac{2}{3}, R$ is 0.19 . If we assume $50 \%$ of the $\psi^{\prime}$ decay to $\psi+$ pions then a similar analysis for the $\psi$ system has $60 \%$ of the $\psi$ particles originating at higher levels and $R=0.05$.

As this work was being completed we became aware of work done by Celmaster, Georgl and Machacek [19] in which some El widths are calculated using a potential denved from data on several lower mass mesons. They used a generalized FermiBreit Hamıltonıan to find triplet splittings, a method which gives splittings for the $\psi$ which are larger than experimental results. Their calculations of El widths are thus the opposite extreme from ours where no splitting was taken into account, ranging from $\frac{1}{2}$ to 3 times our results.

I would like to thank Robert Cahn and Gordon Kane for suggesting and helping with this work and David Rubin for a computer program. 


\section{Note added in proof}

The assumption that $M \approx 2 m$ hals no theoretical basis In fact the $\psi$ data fit better

with $2 m<M$ The correction can be made by ieplacing $m$ by $\frac{1}{2} M$ in eqs (28), (32). (35a), (36a), (37a)

\section{References}

[1] W.R. Innes et al., Phys. Rev. Lett. 39 (1977) 1240.

[2] E. Eichten, K. Gottfried, T Kinoshta, J. Kogut, K D. Lane and T-M. Yan, Phys Rev Lett. 34 (1975) 369 , B.J Harrıngton, S.Y. Park and A Yıldız, Phys. Rev Lett. 34 (1975) 168, 706, J.S. Kang and H.J. Schnitzer, Phys. Rev. D 12 (1975) 841.

[3] E. Eichten and K. Gottfried, Phys. Lett 66B (1977) 286.

[4] C. Quigg and J.L. Rosner, Phys. Lett. 71 B (1977) 153.

[5] M. Mdchacek and Y. Tomozawa, Prog. Theor Phys 58 (1977) 1890, Ann. of Phys. 110 (1978) 407.

[6] W. Celmaster and T.S. Henyey, The Quark-antiquark interaction at all momentum transfers, UC San Diego preprint.

[7] C.T. Bıddıck et dl., Phys. Rev, Lett. 38 (1977) 1324.

[8] G.J. Feldman and M.L. Perl, Phys. Reports 33 (1977) 287

[9] V. Luth et al., Phys. Rev. Lett 35 (1975) 1124

[10] A.M. Boyarskı et al., Phys. Rev. Lett. 34 (1975) 1357.

[11] F. Cichten, K. Gottfried, T. Kinoshita, K D Lane and T-M. Yan, Phys, Rev. Lett. 36 (1976) 500.

[12] R.N. Cahn and S D. Ellis, Decays of the $\Upsilon^{\prime}$ and the structure of resonances at $10 \mathrm{GeV}$, Phys. Rev. D, to be published.

[13] K. Gottfried, The spectroscopy of the new particles, 1977 Int. Symp. on Lepton and Photon Interactions at High Encrgies, Hamburg, Aug. 1977, K. Gottfried, Phys. Rev Lett. 40 (1978) 598.

[14] J.D. Jackson, Lectures on the new particles, in Proc Summer Institute of Particle Physics, SLAC, Stanford CA (1976)

[15] T Appelquist and H D. Politzer, Phys. Rev. Lett. 34 (1975) 43.

[16] R Barbier1, R. Gatto and R. Kogerler, Phys Lett. 60B (1976) 183.

[17] R. Barbier1, R Gatto and E. Remıdd, Phys. Lett. 61B (1976) 465.

[18] Explicit formulae for the 6\% symbols needed here are given by E.P Wigner, "Group Theory" Acad, Press (1959) p 302.

[19] W. Celmaster, H. Georg1 and M. Machacek, Phys. Rev D17 (1978) 886. 